
\title{
25 Research Square \\ Pharmacokinetic and metabolomic studies with a BIO 300 Oral Powder formulation in nonhuman primates
}

\section{Yaoxiang Li}

Georgetown University Medical Center

\section{Michael Girgis}

Georgetown University Medical Center

\section{Meth Jayatilake}

Georgetown University Medical Center

Artur A. Serebrenik

Humanetics Corporation

\section{Amrita K Cheema}

Georgetown University Medical Center

\section{Michael D. Kaytor}

Humanetics Corporation

\section{Vijay K. Singh ( $\nabla$ vijay.singh@usuhs.edu )}

Uniformed Services University of the Health Sciences

\section{Research Article}

Keywords: BIO 300, biomarkers, genistein, pharmacokinetics, nonhuman primates, metabolomics, and radiation countermeasure

Posted Date: February 15th, 2022

DOI: https://doi.org/10.21203/rs.3.rs-1315244/v1

License: (c) (i) This work is licensed under a Creative Commons Attribution 4.0 International License. Read Full License 


\section{Abstract}

$\mathrm{BIO} 300$, a nanosuspension of genistein, is a radiation countermeasure being developed to treat hematopoietic acute radiation syndrome (H-ARS) and the delayed effects of acute radiation exposure (DEARE). Several studies have affirmed its safety and efficacy in alleviating the damaging effects of ionizing radiation. However, dose optimization of any drug has always been an important area of research because unnecessarily high drug doses may result in serious complications. In this study, we assessed the pharmacokinetics (PK) and metabolic profiles of two different doses of BIO 300 Oral Powder $(100 \mathrm{mg} / \mathrm{kg}$ and $200 \mathrm{mg} / \mathrm{kg}$ ) when administered orally to nonhuman primates (NHPs). While the $T_{\max }$ values of both doses remained the same, the area under the curve at $48 \mathrm{~h}\left(\mathrm{AUC}_{0-48}\right)$ was tripled by doubling the dose. Additionally, we monitored serum samples for global metabolomic/lipidomic changes using high resolution mass spectrometry followed by functional pathway analysis prior to and at various time points up to $48 \mathrm{~h}$ post drug administration. Interestingly, the metabolomic profiles of sera from NHPs that received the lower dose demonstrated a transient perturbation in numerous metabolites between the 4 and $12 \mathrm{~h}$ time points. Eventually, the metabolite abundance reverted to near-normal by $48 \mathrm{~h}$. These study results are consistent with our previous studies focused on the PK and metabolomic analysis for parenteral and oral suspension formulations of BIO 300. This study affirms that administration of a single dose of up to $200 \mathrm{mg} / \mathrm{kg}$ of BIO 300 Oral Powder conferred no metabolomic-mediated safety features, suggesting the drug can be safely used to combat radiation injury.

\section{Introduction}

Nuclear accidents and threats can impose a catastrophic impact worldwide ${ }^{1,2}$. In the past few years, numerous US agencies have received nuclear threats that could potentially jeopardize national security and public health. Acute radiation syndrome (ARS) is a pathophysiological condition that is triggered upon exposure to ionizing radiation ${ }^{3-5}$. ARS encompasses 3 sub-syndromes: hematopoietic (H-ARS), gastrointestinal (GI-ARS), and central nervous system (CNS-ARS). CNS-ARS is the major cause of death within days following exposure to high doses of ionizing radiation ${ }^{6-10}$. Total- or partial-body radiation exposures of $\geq 2$ Gray (Gy) are associated with H-ARS and GI-ARS ${ }^{11}$. To date, the United States Food and Drug Administration (US FDA) has approved Neupogen (granulocyte-colony stimulating factor, G-CSF), Neulasta (PEGylated G-CSF), Leukine (granulocyte-macrophage colony-stimulating factor, GM-CSF), and Nplate (romiplostim) for the treatment of H-ARS ${ }^{12-23}$. However, these drugs have safety and therapeutic concerns and require parenteral administration shortly after radiation exposure (24 to $48 \mathrm{~h}$ ), which is not optimal for administration in austere environments. These agents function as cellular growth factors, and their mechanisms of action only support treatment and not prophylaxis for H-ARS.

Genistein (5,7-dihydroxy-3-(4-hydroxyphenyl)chromen-4-one), one of the most abundant isoflavones, was thoroughly examined for its ability to mitigate radiation-induced cellular damage ${ }^{24}$ and although its mechanism of action as a radioprotector is not fully understood, it has selective agonistic activity for estrogen receptor beta $(E R \beta)^{25,26}$. ER $\beta$ operates as a negative feedback mechanism by stimulating cell 
cycle checkpoints and suppressing cell growth ${ }^{27-30}$. Stimulation of ER $\beta$ by genistein has been shown to occur at nanomolar concentrations with an $\mathrm{IC}_{50}$ of $8.4 \mathrm{nM}$. The cell proliferation rate is directly linked to the radiosensitivity. Hence, genistein administration reduces the cell growth rate and enhances radioresistance. Genistein protects against radiation-induced acute myeloid injury when administered prior to irradiation ${ }^{31}$. Furthermore, genistein also arrests hematopoietic stem cells at the G2/M phase and reduces the harmful effects of irradiation ${ }^{32-34}$. Genistein's antioxidant properties also justify its ability to directly scavenge reactive oxygen species (ROS) that are implicated in the formation of cellular oxidative damage including DNA double strand breaks ${ }^{35,36}$.

$\mathrm{BIO} 300$, a nanosuspension formulation of genistein, is a potential radiation countermeasure being developed by Humanetics Corporation and is currently under investigation for the prevention of ARS and the treatment of the delayed effects of acute radiation exposure (DEARE). This agent has also been investigated as a mitigator of lethal radiation-induced pneumonitis/fibrosis in a well-established murine model of whole thorax lung injury. BIO 300 significantly improved survival compared to untreated animals when dosing was initiated $24 \mathrm{~h}$ post-exposure $\left(11 \mathrm{~Gy}, \mathrm{LD}_{50 / 180}\right.$ or $\left.12.5 \mathrm{~Gy}, \mathrm{LD}_{90 / 180}\right)$ and continued once a day for $4-6$ weeks $^{34}$. A single dose of BIO $300(200 \mathrm{mg} / \mathrm{kg})$ administered subcutaneously ( $(s c)$ or intramuscularly $(\mathrm{im}) 24 \mathrm{~h}$ prior to total-body irradiation (TBI) significantly improved survival in mice ${ }^{24}$. When administered by im injection $24 \mathrm{~h}$ prior to lethal TBI, BIO 300 has been shown to attenuate radiation-induced proinflammatory markers (IL-1 $\beta, \mathrm{IL}-6$, and COX-2) in mouse bone marrow and spleen ${ }^{37}$. This suggests that mitigation of the radiation-induced inflammatory response may be a component of the drug's mechanism of action. In addition, we have reported proteomic alterations suggesting that BIO 300 may act through an anti-inflammatory pathway by hampering the complement system or by thrombospondin 1. Additional findings demonstrated that BIO 300 promotes actin elevation ${ }^{10}$.

Currently, BIO 300 is being investigated in nonhuman primates (NHPs) for its efficacy against radiationinduced H-ARS and DEARE. This agent is being developed for regulatory approval following the US FDA Animal Rule. Multiple formulations of BIO 300 have been developed for oral and parenteral routes of administration. BIO 300 Injectable Suspension (BIO 300 IS) is administered through parenteral route, while BIO 300 Oral Suspension (BIO 300 OS) is administered orally (po). BIO 300 OS has demonstrated excellent oral bioavailability in both mice ${ }^{38}$ and $\mathrm{NHPs}^{33}$. Additionally, there is also an oral powder formulation under development. This oral formulation, BIO 300 Oral Powder (BIO 300 OP), is produced by hot-melt extrusion. Our initial study using BIO 300 OP formulation has demonstrated that it has efficacy in a murine H-ARS model equivalent to that of BIO $300 \mathrm{OS}^{24,39}$. The purpose of this study was to determine the pharmacokinetics (PK) of a single dose of BIO $300 \mathrm{OP}$ and the associated metabolic changes in an NHP model. Two oral doses of BIO 300 OP, $100 \mathrm{mg} / \mathrm{kg}$ and $200 \mathrm{mg} / \mathrm{kg}$, were used and blood was drawn over the course of a 48-h period for PK and metabolomics. PK parameters were examined for the most pharmacologically active form of the drug (genistein-aglycone). Serum samples were analysed using global metabolomic and lipidomic profiling (Figure 1). Our results demonstrate modest changes in metabolomic and lipidomic profiles that were transient in nature and reverted to near 
normal levels over a $12 \mathrm{~h}$ period. These findings support the safety of the drug for medical countermeasure use in the field as a radioprotectant or in the clinic as a treatment.

\section{Results}

In this study, we examined the PK parameters of the BIO 300 OP formulation and changes in the metabolomic profile as part of its safety and toxicity assessment in sera of an NHP model. Global metabolomics approaches were utilized with an ultra-performance liquid chromatography quadrupole time-of-flight mass spectrophotometry (UPLC QTOF-MS) platform. We validated the most significant 100 features that showed transient alterations using tandem mass spectrometry and performed pathway analysis on all detected features.

\section{PK parameters of BIO 300 OP was comparable to mouse models but with enhanced bioavailability}

This is the first PK study of BIO 300 OP in a large animal model. A single dose of BIO 300 OP (100 or 200 $\mathrm{mg} / \mathrm{kg}$ ) was administered by oral gavage to NHPs ( $\mathrm{n}=4 / \mathrm{dose})$. The dosing of all four animals was eventless and a single oral gavage administration of BIO 300 OP at a dose of $100 \mathrm{mg} / \mathrm{kg}$ or $200 \mathrm{mg} / \mathrm{kg}$ was considered well tolerated, and no toxicities were observed. First, animals received $100 \mathrm{mg} / \mathrm{kg}$ and then after a washout period of 5 days, the same animals were administered $200 \mathrm{mg} / \mathrm{kg}$. PK parameter evaluation revealed a dose-dependent increase in drug serum level $\left(C_{\max }\right.$ and $\left.A U C\right)$ by doubling the dose (Table 1). The entire drug serum profile is represented in dose response curves versus the time domain (Figure 2). Error bars in the $200 \mathrm{mg} / \mathrm{kg}$ group between $0.25-1 \mathrm{~h}$ are largely due to a single male animal which exhibited genistein-aglycone concentrations $>4 \mathrm{X}$ higher than the average of the other 3 animals. Additionally, no appreciable sex differences were observed between males and females in the $100 \mathrm{mg} / \mathrm{kg}$ group; however, males exhibited a higher $\mathrm{C}_{\max }$ at $200 \mathrm{mg} / \mathrm{kg}$ compared to females, while females had a higher AUC at $200 \mathrm{mg} / \mathrm{kg}$ compared to males (Table 1). This is likely due to natural variation between animals that is amplified due to the low number of animals per group ( $n=2 / \operatorname{sex}$ ). We have previously made similar observations with the PK of BIO 300 OS and BIO 300 IS in NHPs ${ }^{33}$. 
Table 1

Summary of BIO 300 Oral Powder single dose PK parameters in NHPs.

\begin{tabular}{|lllllll|}
\hline Dose & Animals & $\begin{array}{l}\mathrm{T}_{\max } \\
\text { (h) }\end{array}$ & $\begin{array}{l}\mathrm{C}_{\max } \\
(\mathrm{ng} / \mathrm{ml})\end{array}$ & $\begin{array}{l}\mathrm{AUC}_{\mathbf{0}-48} \\
(\mathrm{ng} \cdot \mathrm{h} / \mathrm{ml})\end{array}$ & $\begin{array}{l}\text { AUC }_{0-\text { inf }} \\
(\mathrm{ng} . \mathrm{h} / \mathrm{ml})\end{array}$ & $\mathrm{T}_{1 / 2}(\mathrm{~h})$ \\
\hline $\begin{array}{l}100 \\
\mathrm{mg} / \mathrm{kg}\end{array}$ & All & $1.0 \pm 0.71$ & $662.8 \pm 329.1$ & $2481 \pm 822.2$ & $2505 \pm 832.2$ & $1.69 \pm 0.17$ \\
\cline { 2 - 7 } & Males & $0.75 \pm 0.35$ & $633.5 \pm 326.0$ & $2201 \pm 1304$ & $2214 \pm 1315$ & $1.56 \pm 0.01$ \\
\cline { 2 - 7 } & Females & $1.25 \pm 1.1$ & $692.0 \pm 463.9$ & $2761 \pm 120.9$ & $2796 \pm 94.2$ & $1.83 \pm 0.11$ \\
\hline $\begin{array}{l}200 \\
\mathrm{mg} / \mathrm{kg}\end{array}$ & All & $1.13 \pm 0.63$ & $2867 \pm 3632$ & $7645 \pm 3937$ & $7649 \pm 3938$ & $1.78 \pm 0.27$ \\
\cline { 2 - 7 } & Males & $0.75 \pm 0.35$ & $4504 \pm 5369$ & $7026 \pm 6672$ & $7030 \pm 6673$ & $1.73 \pm 0.46$ \\
\hline Results mean $\pm \mathrm{SD}$ & & & & & $8268 \pm 678.2$ & $1.84 \pm 0.01$ \\
\hline
\end{tabular}


Table 2

Linear mixed effects model showing the abundance of annotated metabolites as the response variable while gender, dose, and time remain the independent variables.

\begin{tabular}{|c|c|c|c|c|c|}
\hline & \multicolumn{5}{|c|}{ Linear Mixed Effect Model from time 1 to $48 \mathrm{~h}$} \\
\hline & & Estimate & Std. Error & t. value & p. value \\
\hline \multirow[t]{4}{*}{ C18(Plasm)-20:4 PE } & (Intercept) & 1.1003 & 0.0812 & 13.5518 & - \\
\hline & Dose & -0.0890 & 0.0646 & -1.3779 & 0.1682 \\
\hline & Gender & -0.0772 & 0.0924 & -0.8357 & 0.4033 \\
\hline & Time* & 0.0050 & 0.0021 & 2.3691 & 0.0178 \\
\hline \multirow[t]{4}{*}{ 16:1 (.DELTA.9-Cis) PC } & (Intercept) & 1.0554 & 0.0580 & 18.2081 & - \\
\hline & Dose* & -0.1222 & 0.0593 & -2.0605 & 0.0393 \\
\hline & Gender & -0.0483 & 0.0593 & -0.8144 & 0.4154 \\
\hline & Time*** & 0.0061 & 0.0019 & 3.2196 & 0.0013 \\
\hline \multirow[t]{4}{*}{ Methyl y-linolenate } & (Intercept) & 0.8299 & 0.0738 & 11.2397 & - \\
\hline & Dose* & 0.1470 & 0.0683 & 2.1523 & 0.0314 \\
\hline & Gender & -0.0620 & 0.0718 & -0.8641 & 0.3875 \\
\hline & Time* & 0.0058 & 0.0025 & 2.3286 & 0.0199 \\
\hline \multirow[t]{4}{*}{ Phosphocholine } & (Intercept) & 0.8759 & 0.0706 & 12.4146 & - \\
\hline & Dose* & 0.1693 & 0.0722 & 2.3451 & 0.0190 \\
\hline & Gender & -0.1060 & 0.0722 & -1.4681 & 0.1421 \\
\hline & Time* & 0.0052 & 0.0023 & 2.2487 & 0.0245 \\
\hline \multirow[t]{4}{*}{ Palmitamide } & (Intercept) & 0.8858 & 0.0746 & 11.8819 & - \\
\hline & Dose* & 0.1642 & 0.0763 & 2.1526 & 0.0314 \\
\hline & Gender & -0.1167 & 0.0763 & -1.5296 & 0.1261 \\
\hline & Time* & 0.0053 & 0.0024 & 2.1608 & 0.0307 \\
\hline \multirow[t]{3}{*}{ Oleic acid } & (Intercept) & 0.8840 & 0.0695 & 12.7141 & - \\
\hline & Dose* & 0.1532 & 0.0712 & 2.1524 & 0.0314 \\
\hline & Gender & -0.1035 & 0.0712 & -1.4552 & 0.1456 \\
\hline
\end{tabular}

A comprehensive list of all detected features with test statistics ( $p$-value, FDR-adjusted $p$-value, fold change and $\log (2)$ fold change) for each tested group post drug administration compared to pre-drug administration for both tested doses. 


\begin{tabular}{|c|c|c|c|c|c|}
\hline & \multicolumn{5}{|c|}{ Linear Mixed Effect Model from time 1 to $48 \mathrm{~h}$} \\
\hline & Time* & 0.0048 & 0.0023 & 2.1200 & 0.0340 \\
\hline \multirow[t]{4}{*}{ Oleoyl ethylamide } & (Intercept) & 0.8499 & 0.0794 & 10.6980 & - \\
\hline & Dose* & 0.1758 & 0.0813 & 2.1619 & 0.0306 \\
\hline & Gender & -0.1560 & 0.0813 & -1.9192 & 0.0550 \\
\hline & Time ${ }^{\star \star \star}$ & 0.0081 & 0.0026 & 3.1088 & 0.0019 \\
\hline \multirow[t]{4}{*}{ Oleamide } & (Intercept) & 0.9410 & 0.1063 & 8.8523 & - \\
\hline & Dose* & 0.3025 & 0.1088 & 2.7805 & 0.0054 \\
\hline & Gender* & -0.2371 & 0.1088 & -2.1799 & 0.0293 \\
\hline & Time* & 0.0079 & 0.0035 & 2.2814 & 0.0225 \\
\hline
\end{tabular}

\section{Administration of $\mathrm{BIO} 300$ leads to transient changes in serum metabolic profiles}

We used high resolution mass spectrometry to analyze longitudinally collected serum samples pre and post BIO 300 administration. Data deconvolution resulted in the detection of approximately 5,000 features. We compared the relative metabolite abundance using statistical analysis to determine temporal patterns over time by comparing baseline samples to post-treatment samples and found modest changes (Supplementary Table 1). Although the higher dose $(200 \mathrm{mg} / \mathrm{kg})$ did not show any significant changes at any time point throughout the study, the lower dose $(100 \mathrm{mg} / \mathrm{kg})$ exhibited temporal changes at the 4 and $8 \mathrm{~h}$ time points which reverted to normal by $12 \mathrm{~h}$.

A principal component analysis (Figure 3, panel A) revealed minimal metabolic changes across all time points except the $4 \mathrm{~h}$ time point. Furthermore, a volcano plot was used to visualize dysregulation of numerous features at the lower dose (Figure 3, Panel B). Statistical analysis performed on all detected features comparing serum level at each time point to the pre-administration time point yielded some significant but transient changes in the metabolomic/lipidomic profile in NHP samples.

The significantly dysregulated features at 4 and $8 \mathrm{~h}$ post BIO 300 treatment were putatively annotated using a database search and subsequently validated by tandem mass spectrometry and matching MS/MS spectra against NIST spectral database (Supplementary Table 2). These included phosphocholines, fatty acids, and fatty acid amides that were downregulated in the 4 - 8-h period (Supplementary Table 3, Figure 4). Furthermore, longitudinal trend line diagrams of each annotated metabolite/lipid throughout the duration of the study were constructed for better visualization of the 
gender and time dependent effects (Figure 5). While C18 (Plasm)-20:4 PE and 16:1 (DELTA 9-CIS) PC were slightly upregulated following either dose $4-12 \mathrm{~h}$ post drug administration, a total of 6 metabolites followed a unique downregulation pattern during a similar time period post drug administration. Taken together, longitudinal analysis of serum metabolomic profiles revealed a transient downregulation in numerous metabolites/lipids at $4-12 \mathrm{~h}$ post drug administration in the serum samples from the NHPs administered the lower dose $(100 \mathrm{mg} / \mathrm{kg})$ compared to the higher dose $(200 \mathrm{mg} / \mathrm{kg})$.

For a comprehensive view of the pathways impacted by the drug administration, we utilized Mummichog 2.06 software (Supplementary Table 4A and B), particularly for the data extracted from the samples at the 4 and $8 \mathrm{~h}$ time points. Pathway analysis at these two time points of interest also revealed association with de novo fatty acid biosynthesis, activation, and metabolism as well as with the TCA cycle and linoleate metabolism. Surprisingly, the lower dose imposed more prominent pathway changes compared to the higher dose. A diagram presents bar charts with all the pathways altered by both tested doses at the 4 and $8 \mathrm{~h}$ time points (Figure 6).

\section{Discussion}

Over the past several years, we have evaluated various BIO 300 formulations for prophylactic dosing against H-ARS in different animal models. While parental formulations were proven safe and effective, it was essential to develop a formulation that is suitable for oral administration in an austere environment, stable at ambient temperature, and highly effective and safe upon administration. To date, the BIO 300 OP formulation appears to satisfy all these requirements. As part of the development of a medical countermeasure, it is imperative to determine the metabolic consequences of drug administration as a measure of safety and efficacy using an NHP model. Herein, we utilized UPLC QTOF-MS based metabolomics and lipidomic profiling to gain insights into metabolic consequences of BIO 300 OP treatment in NHPs. We found transient alterations in phenylalanine, tyrosine, glycerophosphocholine, and glycerophosphoserine after $4 \mathrm{~h}$ of drug treatment that reverted to near pre-treatment levels within $36-48$ $\mathrm{h}$ after drug administration. In addition, there was an overlap in the metabolite classes including palmitamide and oleamide measured in sera from these data compared to that obtained from NHPs administered an im dose of BIO 300 IS and an equivalent dose of BIO 300 OS that we have reported previously ${ }^{33}$. This initial assessment highlighted the importance of using various bioanalytical techniques to interrogate underlying pharmacological actions of the drug and predict drug toxicity. Of note, we did not observe significant changes in the metabolic profile following a single BIO $300 \mathrm{im}$ dose of $200 \mathrm{mg} / \mathrm{kg}$. In part, this could be due to the small number of animals ( 2 males and 2 females) or the modulation of regulatory pathways following the higher dose of BIO 300 OP that abrogate the signals observed following the lower BIO 300 OP dose. The intended dosing regimen for BIO 300 OP is 6 days of consecutive prophylactic dosing; thus, it will be important to analyse the metabolic profile following 6 days of consecutive BIO 300 OP administration in future studies.

While PK parameters for a medical countermeasure candidate are very crucial for a better understanding of the mechanisms of absorption, distribution, and elimination of the drug, dose optimization remains a 
determining factor for the FDA approval process. Analysis of the PK data, obtained from measuring the serum levels of the drug throughout the study for both tested doses, indicate that there was no influence of the dose on the time to maximum plasma concentration $\left(T_{\max }\right)$. Also, the time for half the maximum concentration $\left(T_{1 / 2}\right)$ remains the same. Interestingly, there is an apparent shorter $T_{\max }$ in males compared to females following either of the BIO 300 OP doses. Although this observation was made in only two animals for each sex, it may be correlated with sex-specific PK parameters. The differential mean gut transit time is shorter in males compared to females which could lead to a faster absorption time in males compared to females ${ }^{40,41}$. If this is the case, the shorter $T_{\max }$ in males may constitute a potential safety factor that requires further investigation. One potential implication of this could be that males may require a different dosing regimen (e.g., more frequent dosing) than females. However, one important limitation in our current study was that the PK profile was only examined in two females and two males at each of the BIO 300 OP doses. Confirmatory studies are required to fully investigate potential sexspecific differences in PK.

The average $C_{\max }$ value between groups was nearly quadrupled by doubling the BIO 300 OP dose, while the AUC was only tripled by doubling the dose. Of note, this observation was driven by a single animal in the BIO 300 OP $200 \mathrm{mg} / \mathrm{kg}$ group. Overall, compared to PK parameters of BIO 300 OS in NHPs, BIO 300 OP demonstrated an enhanced bioavailability. At an equivalent dose $(100 \mathrm{mg} / \mathrm{kg}), \mathrm{BIO} 300 \mathrm{OP}$ had a $2 \mathrm{X}$ higher $\mathrm{C}_{\max }$ and 1.3X higher AUC compared to BIO $3000^{33}$. Interestingly, BIO 300 OP appears to be absorbed faster and eliminated more rapidly compared to BIO 300 OS, as evidenced by the 1-h shorter $\mathrm{T}_{\max }$ and the roughly 1 -h shorter half-life $\left(\mathrm{T}_{1 / 2}\right)^{33}$.

Both OP doses resulted in very similar metabolomic profiles compared to the pre-dose samples. However, a slight difference between the tested doses was observed in the $4 \mathrm{~h}$ and later time points. We found transient downregulation of several metabolites including methyl linolenate, phosphocholine, palmitamide, oleic acid, oleoyl ethylamide, and oleamide. This pattern was comparable to our previous finding in which we reported a transient perturbation around the $4 \mathrm{~h}$ time point in the metabolic profiles of C16 sphinganine following the administration of an equivalent BIO 300 OS dose and of glutamate, suberic acid, sphingosine-1-phosphate as well as nonandioic acid following administration of a single BIO 300 IS dose ${ }^{33}$. This could be due to a natural rebound effect caused by the drug's elimination from the blood. This temporal effect was transient and was not correlated with the identification of any safetyrelated features.

A few metabolites were modestly upregulated after administration of the BIO 300 OP formulation including C18(Plasm)-20:4 PE and 16:1 (DELTA 9-CIS) PC. Meanwhile, in our earlier PK and metabolomic studies with BIO 300 when administered orally and $i m$, we observed a slight upregulation around the same time in taurocholic acid and tauroursodeoxycholic acid which appeared after a single im dose of the drug ${ }^{33}$. This could be attributed to transient changes in lipid biosynthesis, activation, and metabolism. These findings were supported by our pathway analysis that we performed on all detected features utilizing Mummichog 2.06v software. Furthermore, statistical analyses built on the annotated metabolites 
indicate that there is a statistically significant difference between the doses of BIO 300 OP evaluated. Also, time seems to be crucial since most of the transient changes appear to revert to baseline by $48 \mathrm{~h}$ post-drug adminitration, which correlates with the clearance of the drug.

Assessment of the bioavailability of the two BIO 300 OP doses demonstrates an expected PK pattern with an expected dose dependent increase in the $C_{\max }$ and AUC values at the higher dose. Longitudinal metabolomic investigations show transient metabolic changes that occur $4-8 \mathrm{~h}$ post drug administration which seem to stabilize over time (by $48 \mathrm{~h}$ ). Moreover, no changes in the metabolome impacted safety and all noted changes were transient in nature, which reaffirms the safety profile and concurs with previously reported preclinical experimental results. BIO 300 is an ideal candidate for further validation assessments as a radiation countermeasure for the prevention of H-ARS.

\section{Material And Methods}

\section{Animals}

Four sex matched NHPs (Macaca mulatta; Chinese sub-strain) weighing $4.0-8.3 \mathrm{~kg}$ and approximately 2 - 5 years old were used in this PK study. Animal study was conducted at Citoxlab, A Charles River Company (Laval, QC, Canada). All animals underwent health assessments prior to inclusion to the study per the test facility standard operating procedures. The study was performed according to the Guide for the Care and Use of Laboratory Animals of the Institute of Laboratory Animal Resources, National Research Council, US National Academy of Sciences ${ }^{42}$. All animal procedures were completed according to a protocol (3019-3873) approved by the Institutional Animal Care and Use Committee of the Citoxlab. This study is reported in accordance with ARRIVE guidelines.

\section{Dosing and serum sample collection from NHPs for PK study}

BIO 300 OP was administered using a gavage tube attached to a syringe, and was delivered as a suspension in vehicle $(0.5 \%(\mathrm{w} / \mathrm{v})$ Methocel A4M and $3 \%(\mathrm{w} / \mathrm{v})$ polyvinylpyrrolidone $\mathrm{K} 25$ in water). The volume of BIO $300 \mathrm{OP}$ administered was calculated and adjusted based on the most recent body weight of each animal. Following dosing, the gavage tube was rinsed with $10 \mathrm{ml}$ water to ensure the entire dose volume was delivered.

A single dose of BIO 300 OP was administered twice by oral gavage to 4 NHPs, first the $100 \mathrm{mg} / \mathrm{kg}$ dose and then the $200 \mathrm{mg} / \mathrm{kg}$ dose in the same 4 animals. Doses were separated by a 5-day wash-out period. Blood samples were collected from all animals at the following targeted time points: pre-dose, at 0.25 , $0.5,1,2,4,8,12,24$, and $48 \mathrm{~h}$ after dosing. Each blood sample was collected by femoral, cephalic, or saphenous vein into serum-separating tubes and allowed to clot at room temperature. Samples were centrifuged under refrigeration (set to $+4^{\circ} \mathrm{C}$ at $1,500 \mathrm{~g}$ ) for $10 \mathrm{~min}$ and allowed to separate, and serum was then collected. 


\section{PK analysis}

Bioanalysis of NHP serum samples to determine non-glucuronidated genistein (free genistein or genistein aglycone; active drug) was conducted by Inotiv (West Lafayette, IN USA), using a LC-MS/MS technique in accordance with the previously described protocol ${ }^{33}$. Serum samples were analyzed to determine genistein aglycone concentrations. Genistein aglycone is thought to be the more active form of the drug compared to its conjugates and is the most direct indicator of drug efficacy ${ }^{43}$. Data acquisition was performed using Analyst software. Regression and calculation of results and statistics were performed using Watson $\circledast$ LIMS v7.5 (Thermo Fisher Scientific, Waltham, MA, USA). Fourteen of the NHP serum samples were confirmed by re-analysis because they fell outside the expected result range based on the analysis of samples from nearby time points. Repeat values were within $5 \%$ of the initial observations.

PK parameters were estimated using nominal sampling times relative to each dose administered and nominal doses unless otherwise specified. Serum concentration values obtained at the pre-dose time point were used to estimate the concentration at time zero whenever possible. Unquantifiable concentration values (BQL) were assigned a value of zero. The AUC vs. time curves were calculated using the linear trapezoidal method with linear interpolation. The AUC was not calculated for PK profiles with less than 3 quantifiable concentrations of the test item at separate time points. When practicable, the terminal elimination phase of each concentration versus time curve was identified using at least the final three observed concentration values after $\mathrm{C}_{\max }$, but not including $\mathrm{C}_{\max }$. The slope of the terminal elimination phase was determined using log linear regression on the unweighted concentration data. Parameters relying on the determination of the terminal elimination phase were not reported if the

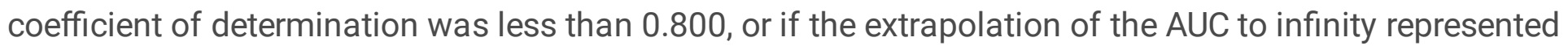
more than $20 \%$ of the total area.

\section{Serum Metabolomics Using UPLC QTOF Analysis}

Metabolite extraction was performed by combining $25 \mu \mathrm{L}$ of serum with $75 \mu \mathrm{L}$ of an extraction solution made up of $35 \%$ water, $25 \%$ methanol, $40 \%$ isopropanol, $0.1 \%$ debrisoquine $\left(1 \mathrm{mg} / \mathrm{ml}\right.$ in $\left.\mathrm{ddH}_{2} \mathrm{O}\right)$ and $0.5 \%$ 4-nitrobenzoic acid ( $1 \mathrm{mg} / \mathrm{ml}$ in methanol). The samples were vortexed and kept at $4^{\circ} \mathrm{C}$ for $20 \mathrm{~min}$. Next, they were combined with $100 \mu \mathrm{L}$ of acetonitrile (ACN) and left at $-20^{\circ} \mathrm{C}$ for $15 \mathrm{~min}$. Finally, the samples were centrifuged at $15,493 \mathrm{xg}$ for $20 \mathrm{~min}$ at $4^{\circ} \mathrm{C}$ and the supernatants were transferred to MS vials for LCMS analysis. A quality control sample (QC) was also prepared by pooling an aliquot of each of the prepared samples and was run periodically throughout the acquisition sequence.

The sample extracts were acquired on an Acquity UPLC coupled to a Xevo G2 QTOF-MS (Waters Corporation, Milford, MA). A volume of $2 \mu \mathrm{L}$ of each sample was injected onto either an Acquity UPLC BEH C18, $130 \AA, 1.7 \mu \mathrm{m}, 2.1 \mathrm{~mm} \times 50 \mathrm{~mm}$ column maintained at $40^{\circ} \mathrm{C}$ for the metabolomics acquisition or a $\mathrm{CSH} C 18,130 \AA, 1.7 \mu \mathrm{m}, 2.1 \mathrm{~mm} \times 100 \mathrm{~mm}$ column maintained at $65^{\circ} \mathrm{C}$ for the lipidomics acquisition. The LC solvents used were $100 \%$ water with $0.1 \%$ formic acid (A), $100 \%$ ACN with $0.1 \%$ formic acid (B), $100 \%$ isopropanol with $0.1 \%$ formic acid and $10 \mathrm{mM}$ ammonium formate (C). The metabolomics gradient 
with a flow rate of $0.5 \mathrm{~m} / \mathrm{min}$ was set as follows: initial $-98 \%$ A, 2\% B; $0.5 \mathrm{~min}-98 \%$ A, 2\% B; 4.0 min $40 \%$ A, 60\% B; 8.0 min - 2\% A, 98\% B; 9.0 min - 2\% B, 98\% D; 9.5 min - 11.2\% B, 88.2\% C; 11.0 min $11 \%$ B, $88.2 \%$ C; $11.5 \mathrm{~min}-50 \%$ A, 50\% B; $12.0 \mathrm{~min}-98 \%$ A, 2\% B, $13.0 \mathrm{~min}-98 \%$ A, $2 \%$ B. The lipidomics solvents were the same as previously mentioned with the addition of $10 \mathrm{mM}$ ammonium formate. The gradient had a flow rate of $0.45 \mathrm{~m} / \mathrm{min}$ and was run set as follows: initial $-30 \% A, 34 \% B$, $36 \%$ C; $0.5 \min -30 \%$ A, 34\% B, 36\% C; 8.0 min - 10\% B, 90\% C; 8.5 min - 10\% B, 90\% C; 9.0 min - 30\% A, $34 \% \mathrm{~B}, 36 \% \mathrm{C}$; and $11.0 \mathrm{~min}-30 \% \mathrm{~A}, 34 \% \mathrm{~B}, 36 \% \mathrm{C}$.

The column eluent was introduced into the QTOF MS operating in either positive or negative by electrospray ionization. Positive mode had a capillary voltage of $3.00 \mathrm{kV}$ and a sampling cone voltage of $30 \mathrm{~V}$. Negative mode had a capillary voltage of $2.00 \mathrm{kV}$ and had a sampling cone voltage of $30 \mathrm{~V}$. The desolvation gas flow was set to $1000 \mathrm{~L} / \mathrm{h}$ and the desolvation temperature was set to $500^{\circ} \mathrm{C}{ }^{44}$. The cone gas flow was $25 \mathrm{~L} / \mathrm{h}$, and the source temperature was $120^{\circ} \mathrm{C}$. The data was acquired in the sensitivity MS mode with a scan time of 0.300 seconds and an interscan time of 0.014 seconds. Accurate mass was maintained by infusing leucine enkephalin $(556.2771 \mathrm{~m} / \mathrm{z})$ in $50 \%$ aqueous acetonitrile $(2.0 \mathrm{ng} / \mathrm{ml})$ at a rate of $20 \mu \mathrm{L} / \mathrm{min}$ via the Lockspray interface every 10 seconds. Data was acquired in Centroid mode with a 50.0 to $1200.0 \mathrm{~m} / \mathrm{z}$ mass range for ToF MS scanning ${ }^{44}$. The pooled QC was injected every 10 samples to monitor any shifts in retention time and intensities.

\section{Data Processing and Statistical Analysis}

Data were log transformed and scaled following feature detection; statistical comparisons were performed to follow longitudinal changes in metabolomics profiles and their possible impact on the phenotype. Untargeted metabolomics raw data files were first converted to the NetCDF file format using the Databridge tool in MassLynx (Waters Corporation, Milford, MA). All parameters for peak picking were optimized by IPO ${ }^{45}$ (Isotopologue Parameter Optimization) R package then processed by XCMS ${ }^{46}$ package. Data was normalized based on the internal standard and QC-RLSC (QC robust LOESS signal correction ${ }^{47}$ ). The level of differential intensities for each metabolite was calculated using paired t-test, comparing each time point after drug administration to pre-administration for each dose (effect of drug) constrained by false discovery rate (FDR)-adjusted $p$ value $<0.05$. The identity of the most significant features was further validated using tandem mass spectrometry by fragmentation pattern matching against National Institute of Standards and Technology (NIST) database. Pathway analysis was performed using the Mummichog software v2.06. To examine the statistical significance of the drastic change in the MS/MS validated metabolites over $48 \mathrm{~h}$ across gender dosage and time, linear mixed effects models were fitted into a log2 transformed metabolite intensities as the response variable, while the gender (categorical variable: female and male), dose (categorical variable: $100 \mathrm{mg} / \mathrm{kg}$ and 200 $\mathrm{mg} / \mathrm{kg}$ ), and time were considered independent variables. All statistical analyses were performed using recruiting SAS software (version 9.4; SAS Institute Inc., Cary; North Carolina, USA) and R (version 4.0.2).

\section{Declarations}


Supplementary Materials:

All the supplementary materials mentioned in this paper can be found at

Author Contributions:

M.D.K., A.A.S., V.K.S., A.K.C. Study design, V.K.S., A.A.S., M.D.K., A.K.C., M.G, Y.L., M.J. Performance of the study, V.K.S., A.A.S., M.D.K., A.K.C., M.G., Y.L., M.J. Drafting of the manuscript. V.K.S., A.A.S., M.D.K, A.K.C., M.G., Y.L. Revised manuscript content. All authors reviewed the manuscript.

Funding:

This study was supported by funding from Congressionally Directed Medical Research Programs grant \# W81XWH-17-1-0584 awarded to MDK (subaward to VKS by MDK/Humanetics Corporation) and Joint Program Committee-7/Radiation Health Effects Research Program grant \# 1180 VP00262-01 awarded and Armed Forces Radiobiology Research Institute/Uniformed Services University of the Health Sciences grant \# AFR-B4-10978 awarded to VKS.

Acknowledgments:

The opinions or assertions contained herein are the private views of the authors and are not necessarily those of the Uniformed Services University of the Health Sciences, or the Department of Defense, USA. The authors would like to acknowledge the Metabolomics Shared Resource in Georgetown University (Washington, DC, USA) which is partially supported by $\mathrm{NIH} / \mathrm{NCl} / \mathrm{CCSG}$ grant P30-CA051008. We are thankful to Ms. Alana Carpenter for editing the manuscript.

Conflicts of Interest:

Dr. Artur A. Serebrenik and Dr. Michael D. Kaytor are employees of Humanetics Corporation, Minneapolis, MN, USA. The other authors report no conflicts of interest. The authors alone are responsible for the content and writing of this paper.

\section{ORCID:}

Vijay K Singh https://orcid.org/0000-0002-6631-3849

Data Availability

All data generated or analyzed during this study are included in this article (and its Supplementary files)

\section{References}

1. Hasegawa, A. et al. Health effects of radiation and other health problems in the aftermath of nuclear accidents, with an emphasis on Fukushima. Lancet 386, 479-488, doi:10.1016/S0140- 
6736(15)61106-0 (2015).

2. Marzaleh Co-First Author, M. A. et al. Design and validation of a hospital emergency department preparedness questionnaire for radiation accidents, nuclear accidents, and nuclear terrorism in Iran. Am J Disaster Med 15, 283-292, doi:10.5055/ajdm.2020.0377 (2020).

3. MacVittie, T. J., Farese, A. M. \& Jackson, W. E., 3rd. A systematic review of the hematopoietic acute radiation syndrome (H-ARS) in canines and non-human primates: acute mixed neutron/gamma vs. reference quality radiations. Health Phys 119, 527-558, doi:10.1097/HP.0000000000001319 (2020).

4. Singh, V. K. \& Olabisi, A. O. Nonhuman primates as models for the discovery and development of radiation countermeasures. Expert Opin Drug Discov 12, 695-709, doi:10.1080/17460441.2017.1323863 (2017).

5. Singh, V. K. \& Seed, T. M. A review of radiation countermeasures focusing on injury-specific medicinals and regulatory approval status: part I. Radiation sub-syndromes, animal models and FDAapproved countermeasures. Int J Radiat Biol 93, 851-869, doi:10.1080/09553002.2017.1332438 (2017).

6. Singh, V. K., Newman, V. L., Berg, A. N. \& MacVittie, T. J. Animal models for acute radiation syndrome drug discovery. Expert Opin Drug Discov 10, 497-517, doi:10.1517/17460441.2015.1023290 (2015).

7. Girgis, M. et al. Short-term metabolic disruptions in urine of mouse models following exposure to low doses of oxygen ion radiation. J Environ Sci Health C Toxicol Carcinog 39, 234-249, doi:10.1080/26896583.2020.1868866 (2021).

8. Li, Y. et al. Analysis of the metabolomic profile in serum of irradiated nonhuman primates treated with Ex-Rad, a radiation countermeasure. Sci Rep 11, 11449, doi:10.1038/s41598-021-91067-9 (2021).

9. Dissmore, T. et al. Longitudinal metabolic alterations in plasma of rats exposed to low doses of high linear energy transfer radiation. J Environ Sci Health C Toxicol Carcinog 39, 219-233, doi:10.1080/26896583.2020.1865027 (2021).

10. Girgis, M. et al. Comparative proteomic analysis of serum from nonhuman primates administered BIO 300: a promising radiation countermeasure. Sci Rep 10, 19343, doi:10.1038/s41598-020-764944 (2020).

11. Hall, E. J. \& Giaccia, A. J. Radiobiology for the Radiobiologist. 7th edn, (Lippincott Williams and Wilkins, 2012).

12. Farese, A. M. \& MacVittie, T. J. Filgrastim for the treatment of hematopoietic acute radiation syndrome. Drugs Today (Barc) 51, 537-548, doi:10.1358/dot.2015.51.9.2386730 (2015).

13. Hankey, K. G. et al. Pegfilgrastim improves survival of lethally irradiated nonhuman primates. Radiat Res 183, 643-655, doi:10.1667/RR13940.1 (2015).

14. Singh, V. K. \& Seed, T. M. An update on sargramostim for treatment of acute radiation syndrome. Drugs Today (Barc) 54, 679-693, doi:10.1358/dot.2018.54.11.2899370 (2018).

15. Zhong, Y. et al. Efficacy of delayed administration of sargramostim up to 120 hours post exposure in a nonhuman primate total body radiation model. Int J Radiat Biol 97, S100-S116, 
doi:10.1080/09553002.2019.1673499 (2021).

16. Clayton, N. P. et al. Sargramostim (rhu GM-CSF) improves survival of non-human primates with severe bone marrow suppression after acute, high-dose, whole-body irradiation. Radiat Res 195, 191-199, doi:10.1667/RADE-20-00131.1 (2021).

17. U.S. Food and Drug Administration. Animal Rule approvals, <https://www.fda.gov/drugs/nda-andbla-approvals/animal-rule-approvals> (2021).

18. Wong, K. et al. Pharmacodynamics of romiplostim alone and in combination with pegfilgrastim on acute radiation-induced thrombocytopenia and neutropenia in non-human primates. Int $\mathrm{J}$ Radiat Biol 96, 155-166, doi:10.1080/09553002.2019.1625488 (2020).

19. Gale, R. P. \& Armitage, J. O. Use of molecularly-cloned haematopoietic growth factors in persons exposed to acute high-dose, high-dose rate whole-body ionizing radiations. Blood Rev 45, 100690, doi:10.1016/j.blre.2020.100690 (2021).

20. Wong, K. et al. in Annual Conference of Radiation Research Society (Virtual, 2020).

21. Farese, A. M. et al. Filgrastim improves survival in lethally irradiated nonhuman primates. Radiat Res 179, 89-100, doi:10.1667/RR3049.1 (2013).

22. Singh, V. K. \& Seed, T. M. Radiation countermeasures for hematopoietic acute radiation syndrome: growth factors, cytokines and beyond. Int J Radiat Biol 97, 1526-1547, doi:10.1080/09553002.2021.1969054 (2021).

23. Singh, V. K. \& Seed, T. M. An update on romiplostim for treatment of acute radiation syndrome. Drugs Today (Barc) (in press) (2022).

24. Singh, V. K. \& Seed, T. M. BIO 300: a promising radiation countermeasure under advanced development for acute radiation syndrome and the delayed effects of acute radiation exposure. Exp Opin Invest Drugs 29, 429-441, doi:10.1080/13543784.2020.1757648 (2020).

25. Landauer, M. R., Harvey, A. J., Kaytor, M. D. \& Day, R. M. Mechanism and therapeutic window of a genistein nanosuspension to protect against hematopoietic-acute radiation syndrome. J Radiat Res 60, 308-317, doi:10.1093/jrr/rrz014 (2019).

26. Landauer, M. in Herbal Radiomodulators: Applications in Medicine, Homeland Defense and Space (ed R. Arora) 163-173 (CABI Publishing, 2008).

27. Haddad, Y. H., Said, R. S., Kamel, R., Morsy, E. M. E. \& El-Demerdash, E. Phytoestrogen genistein hinders ovarian oxidative damage and apoptotic cell death-induced by ionizing radiation: cooperative role of ER-beta, TGF-beta, and FOXL-2. Sci Rep 10, 13551, doi:10.1038/s41598-020-703092 (2020).

28. Prossnitz, E. R. \& Barton, M. The G-protein-coupled estrogen receptor GPER in health and disease. Nat Rev Endocrinol 7, 715-726, doi:10.1038/nrendo.2011.122 (2011).

29. Xu, L., Liu, J. T., Li, K., Wang, S. Y. \& Xu, S. Genistein inhibits Ang Il-induced CRP and MMP-9 generations via the ER-p38/ERK1/2-PPARgamma-NF-kappaB signaling pathway in rat vascular smooth muscle cells. Life Sci 216, 140-146, doi:10.1016/j.Ifs.2018.11.036 (2019). 
30. Li, X. L. et al. Differential effects of genistein and 8-prenylgenistein on reproductive tissues in immature female mice. Pharm Biol 57, 226-230 (2019).

31. Day, R. M. et al. Enhanced hematopoietic protection from radiation by the combination of genistein and captopril. Int Immunopharmacol 15, 348-356, doi:10.1016/j.intimp.2012.12.029 (2013).

32. Jackson, I. L. et al. BIO 300, a nanosuspension of genistein, mitigates radiation-induced erectile dysfunction and sensitizes human prostate cancer xenografts to radiation therapy. Int J Radiat Oncol Biol Phys 105, 400-409, doi:10.1016/j.ijrobp.2019.05.062 (2019).

33. Cheema, A. K. et al. Pharmacokinetic and metabolomic studies with BIO 300, a nanosuspension of genistein, in a nonhuman primate model. Int J Mol Sci 20, 1231, doi:10.3390/ijms20051231 (2019).

34. Jackson, I. L. et al. BIO 300, a nanosuspension of genistein, mitigates pneumonitis/fibrosis following high-dose radiation exposure in the C57L/J murine model. Br J Pharmacol 174, 4738-4750, doi:10.1111/bph.14056 (2017).

35. Park, C. et al. Induction of G2/M cell cycle arrest and apoptosis by genistein in human bladder cancer T24 cells through inhibition of the ROS-dependent PI3k/Akt signal transduction pathway. Antioxidants 8, doi:10.3390/antiox8090327 (2019).

36. Zhang, Q., Bao, J. \& Yang, J. Genistein-triggered anticancer activity against liver cancer cell line HepG2 involves ROS generation, mitochondrial apoptosis, G2/M cell cycle arrest and inhibition of cell migration. Arch Med Sci 15, 1001-1009, doi:10.5114/aoms.2018.78742 (2019).

37. Ha, C. T., Li, X. H., Fu, D., Xiao, M. \& Landauer, M. R. Genistein nanoparticles protect mouse hematopoietic system and prevent proinflammatory factors after gamma irradiation. Radiat Res 180, 316-325, doi:10.1667/RR3326.1 (2013).

38. Salem, A. M. et al. Interspecies comparison and irradiation effect on pharmacokinetics of BIO 300, a nanosuspension of genistein, following different routes of administration in mice and non-human primates. Radiat Res (in press) (2022).

39. Singh, V. K. et al. A novel oral formulation of BIO 300 confers prophylactic radioprotection from acute radiation syndrome in mice. Int J Radiat Biol (in press), doi:10.1080/09553002.2021.1981556 (2022).

40. Soldin, O. P. \& Mattison, D. R. Sex differences in pharmacokinetics and pharmacodynamics. Clin Pharmacokinet 48, 143-157, doi:10.2165/00003088-200948030-00001 (2009).

41. Caballeria, J., Baraona, E., Rodamilans, M. \& Lieber, C. S. Effects of cimetidine on gastric alcohol dehydrogenase activity and blood ethanol levels. Gastroenterology 96, 388-392, doi:10.1016/00165085(89)91562-x (1989).

42. National Research Council of the National Academy of Sciences. Guide for the care and use of laboratory animals. 8th edn, (National Academies Press, 2011).

43. Yang, Z., Kulkarni, K., Zhu, W. \& Hu, M. Bioavailability and pharmacokinetics of genistein: mechanistic studies on its ADME. Anticancer Agents Med Chem 12, 1264-1280, doi:10.2174/187152012803833107 (2012). 
44. Cheema, A. K. et al. Microbiome study in irradiated mice treated with $\mathrm{BIO} 300$, a promising radiation countermeasure. Anim Microbiome 3, 71, doi:10.1186/s42523-021-00132-1 (2021).

45. Libiseller, G. et al. IPO: a tool for automated optimization of XCMS parameters. BMC bioinformatics 16, 118, doi:10.1186/s12859-015-0562-8 (2015).

46. Smith, C. A., Want, E. J., O'Maille, G., Abagyan, R. \& Siuzdak, G. XCMS: processing mass spectrometry data for metabolite profiling using nonlinear peak alignment, matching, and identification. Anal Chem 78, 779-787, doi:10.1021/ac051437y (2006).

47. Dunn, W. B. et al. Procedures for large-scale metabolic profiling of serum and plasma using gas chromatography and liquid chromatography coupled to mass spectrometry. Nat Protoc 6, 10601083, doi:10.1038/nprot.2011.335 (2011).

\section{Figures}

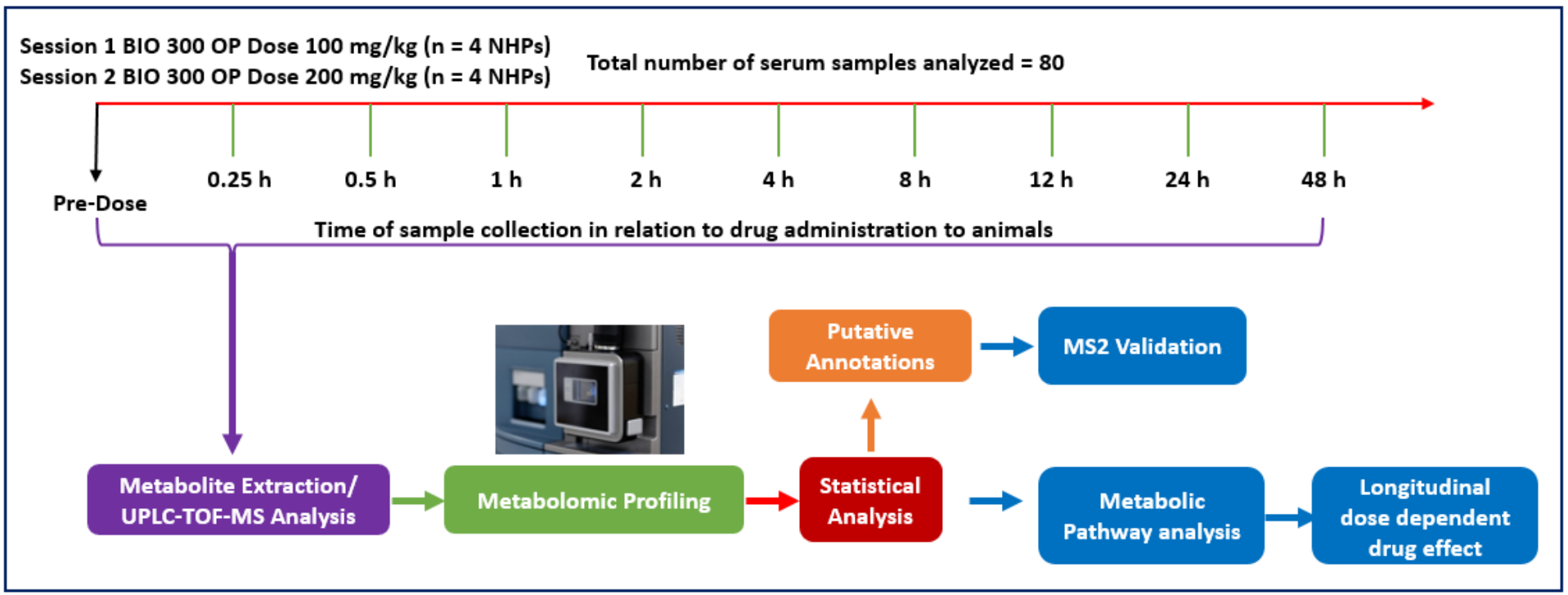

\section{Figure 1}

Overall study design schema for metabolic and PK examination following BIO 300 Oral Powder administration to NHPs. 


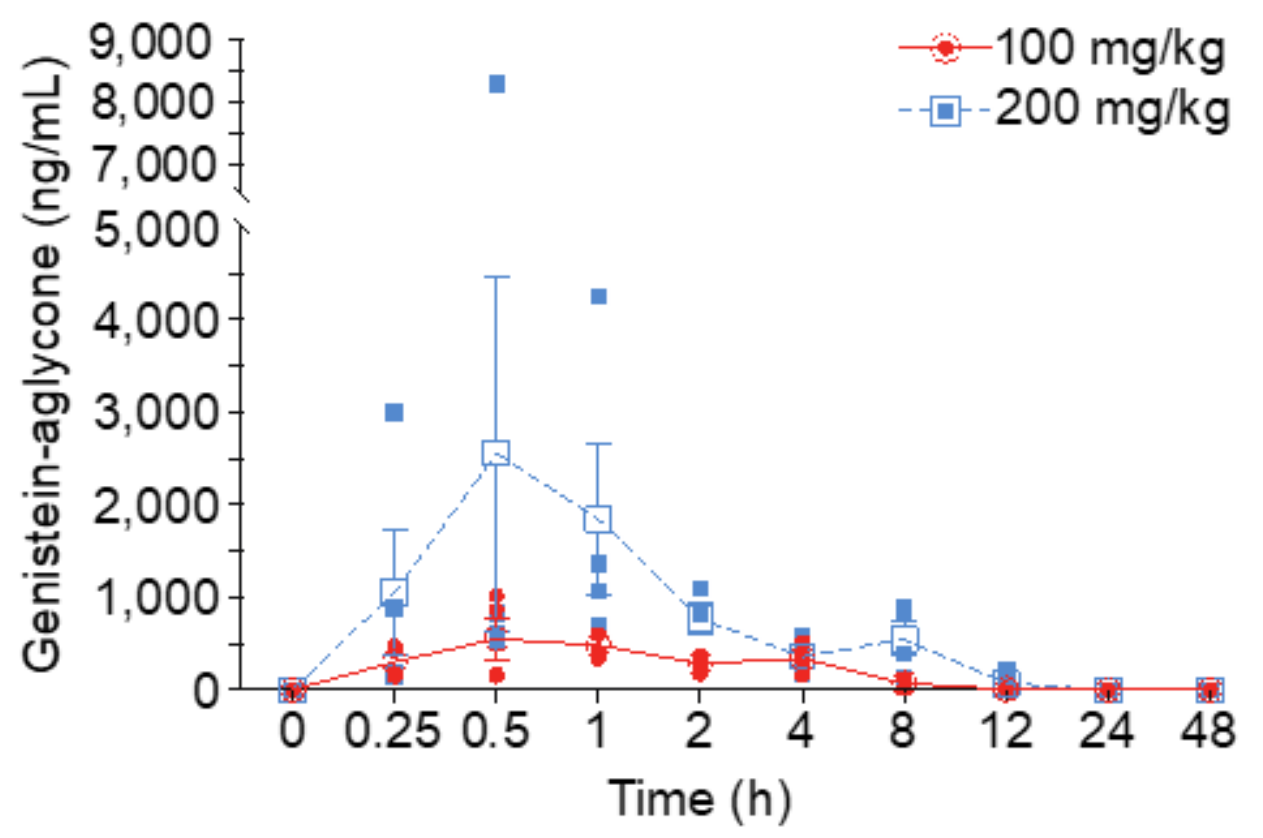

Figure 2

Pharmacokinetic analysis of BIO 300 Oral Powder (OP) formulation for two doses $(100 \mathrm{mg} / \mathrm{kg}$ and 200 $\mathrm{mg} / \mathrm{kg}$ ). Open Symbols are the average of $\mathrm{N}=4 \mathrm{NHPs}$. Closed symbols are the individual NHP genisteinaglycone concentrations for the color-matched dosing groups. Error bars are standard error, and they are not shown when the error bars are smaller than the symbol. 


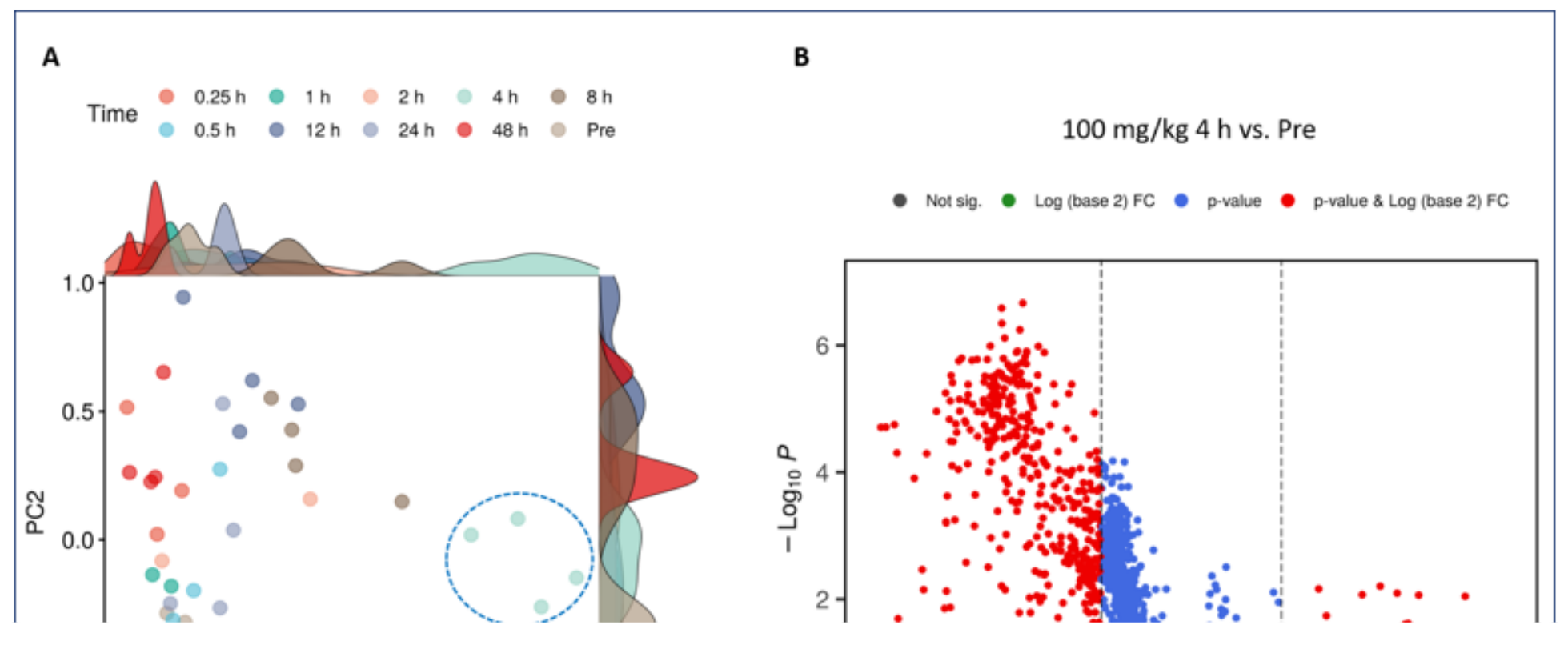

Figure 3

A 2D-PCA plot showing time dependent group separation for the lower dose of $\mathrm{BIO} 300$ Oral Powder (100 $\mathrm{mg} / \mathrm{kg}$ ) (Panel A). Volcano plot showing dysregulated metabolites $4 \mathrm{~h}$ after BIO 300 Oral Powder administration (panel B).

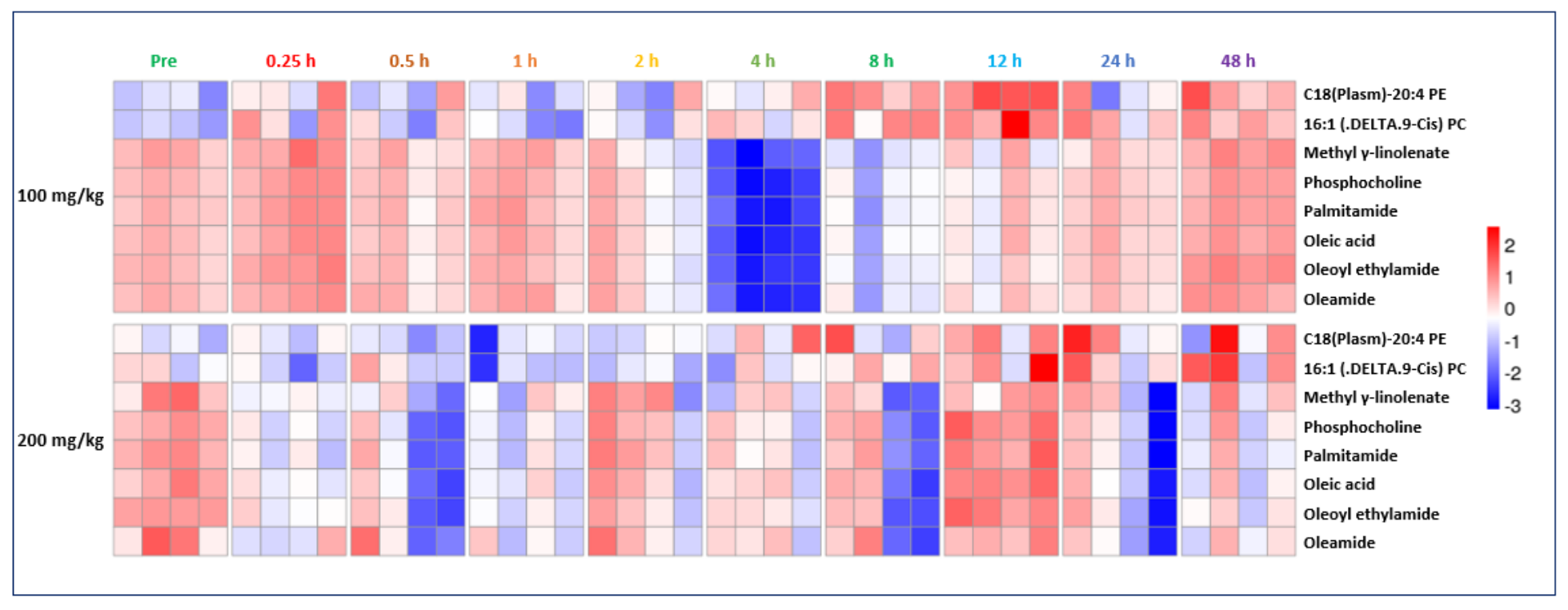

\section{Figure 4}

Hierarchical clustering based heatmap visualization of changes in serum abundance of annotated metabolites and lipids over time. 


\section{Figure 5}

A trend line of the performance of a subset of metabolites/lipids over the study time course showing transient changes that stabilize over time.

A

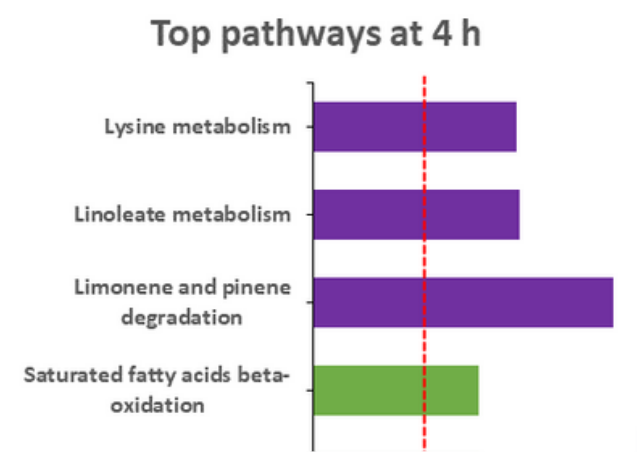

B

Top pathways at $8 \mathrm{~h}$

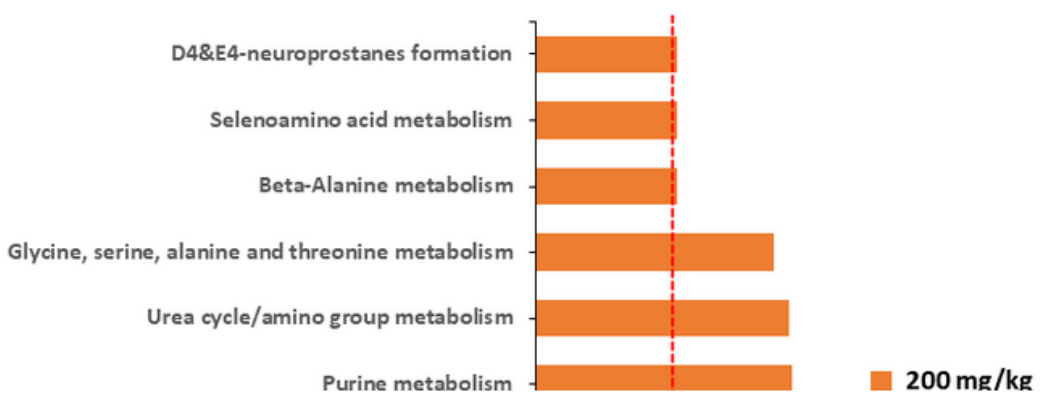

\section{Figure 6}

Pathway analysis as computed utilizing all detected features of both tested doses at the $4 \mathrm{~h}$ as well as the $8 \mathrm{~h}$ time points using Mummichog 2.06 software (panels A and B). The dotted lines denote the significance threshold.

\section{Supplementary Files}

This is a list of supplementary files associated with this preprint. Click to download.

- 20220202CombinedSupplfile.pdf 